\title{
Noiseless non-reciprocity in a parametric active device
}

\author{
Archana Kamal', John Clarke² and M. H. Devoret ${ }^{1 \star}$
}

Non-reciprocal devices such as circulators and isolators belong to an important class of microwave components employed in applications including the measurement of mesoscopic circuits at cryogenic temperatures ${ }^{1-5}$. The measurement protocols usually involve an amplification chain that relies on circulators to separate input and output channels and to suppress backaction from different stages on the sample under test. In these devices the usual reciprocal symmetry of circuits is broken by the phenomenon of Faraday rotation based on magnetic materials and fields ${ }^{6}$. However, magnets are averse to on-chip integration, and magnetic fields are deleterious to delicate superconducting devices ${ }^{7,8}$. Here we present a new proposal that combines two stages of parametric modulation to emulate the action of a circulator. It is devoid of magnetic components and suitable for on-chip integration. As the design is free of any dissipative elements and based on reversible operation, the device operates noiselessly, giving it an important advantage over other non-reciprocal active devices for quantum information processing applications.

Reciprocity is one of the fundamental symmetries frequently encountered in electrical circuits. It is equivalent to the more familiar notion of the principle of reversibility in optics, which states that any experiment is symmetric under an exchange of source and image ${ }^{9}$. Reciprocity can, however, be violated, for example, by the magneto-optic Faraday effect ${ }^{6}$ - the rotation of the polarization vector of light resulting from different propagation velocities of left- and right-circularly polarized waves in the presence of an applied magnetic field $\mathbf{B}$ parallel to the direction of propagation (Fig. 1). The non-reciprocal phenomenon of Faraday rotation should be contrasted with the superficially similar, though reciprocal, effect of optical activity where the polarization vector of light is rotated on passage through a non-centrosymmetric (chiral) medium. This change in the sense of rotation of polarization for counterpropagating waves in a Faraday medium (as seen by the observer receiving the light) has led some physicists to refer to this effect as a form of time-reversal symmetry breaking, a use of words that we prefer to avoid here ${ }^{10}$.

The phenomenon of non-reciprocity has propelled numerous theoretical investigations (ref. 11 and references therein); furthermore, it offers immediate practical applications. Recent progress in solid-state superconducting qubits, which provide some of the most promising architectures for scalable quantum computers ${ }^{12,13}$, has generated a strong incentive to integrate the components required for qubit operations and readout on-chip for incorporation in future quantum mechanical processors. A large variety of qubit readout protocols involve microwave-reflection-based measurements and rely on non-reciprocal devices such as circulators (or isolators) for separation of input and output channels ${ }^{3,5,14}$. These devices also play a strategic role in measurements based

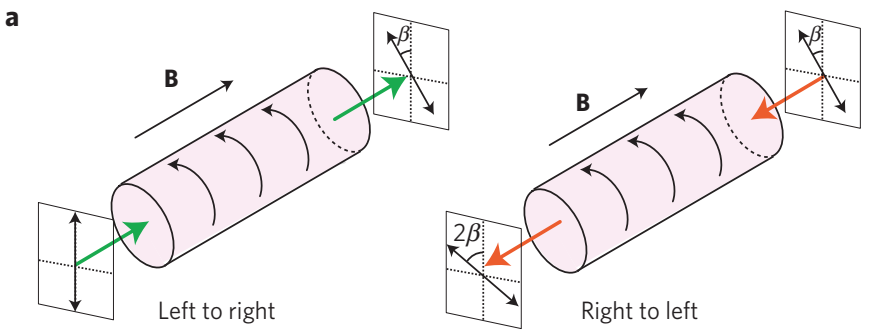

b

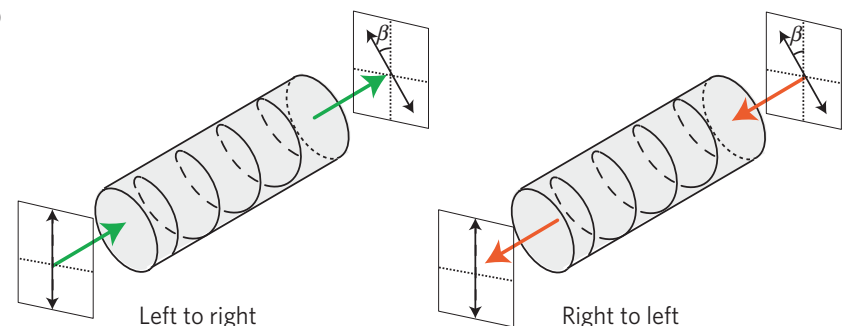

c
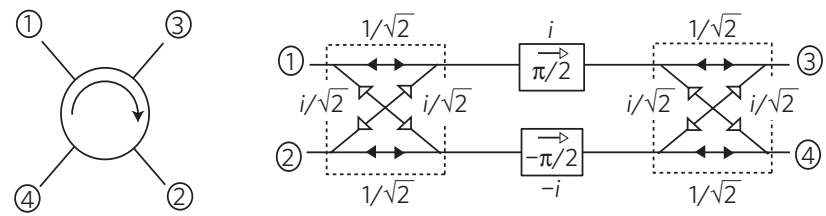

Figure 1 | Faraday rotation and circulator action. a, Faraday rotation for a wave travelling from left to right in a Faraday-active medium, followed by a reflection back into the medium leading to a reversal of the direction of propagation. The rotation of the light polarization is fixed to a rotation-like property of the medium (shown by the arrows), set by an external magnetic field oriented along the propagation axis. The sense of light rotation as seen with respect to the direction of propagation remains the same, leading to the doubling of the rotation angle on reversing the ray through the medium. b, Rotation of the polarization vector of light on passage through an optically active medium, on the other hand, cancels out on reversing the direction of propagation. This occurs because optical rotation depends on the chirality of the medium (represented as a helix), which also reverses with the direction of propagation. $\mathbf{c}$, Representation and schematic design of a conventional four-port circulator. The device consists of two $90^{\circ}$

hybrids (equivalent to optical beam splitters) separated by a non-reciprocal phase shifter based on Faraday rotation. The filled black arrows indicate an amplitude split with no phase change and the open arrows indicate an amplitude split with a $90^{\circ}$ phase change. The non-reciprocal phase shift is effective only for the propagation direction indicated by the arrow on the phase-shifter rectangle.

${ }^{1}$ Department of Physics and Applied Physics, Yale University, 15 Prospect Street, New Haven, Connecticut 06520, USA, ${ }^{2}$ Department of Physics, University of California and Materials Sciences Division, Lawrence Berkeley National Laboratory, Berkeley, California 94720, USA. *e-mail: michel.devoret@yale.edu. 
a

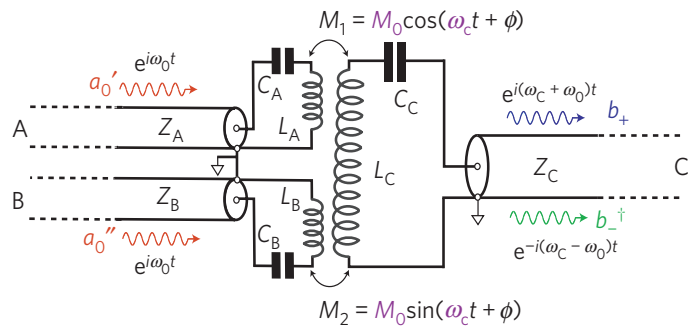

b

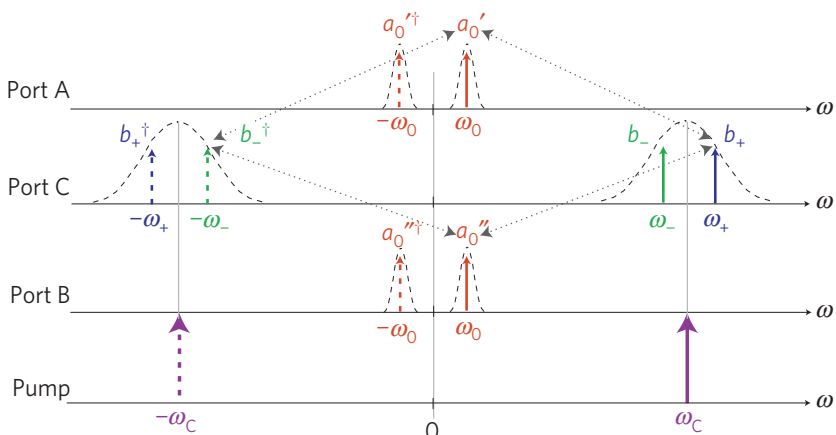

Figure 2 | Description of an active reversible (information-conserving) in-phase/quadrature modulator carrying out frequency UDC. a, Circuit schematic of the UDC containing only dispersive components. The two low-frequency series $L C$ resonators (with $L_{A}=L_{B}$ and $C_{A}=C_{B}$ ) are fed by two input semi-infinite transmission lines, $A$ and $B$, and parametrically coupled to a third high-frequency series $\mathrm{LC}$ resonator leading to an output line $\mathrm{C}$. The parametric coupling is achieved by varying the mutual inductances $M_{1}$ and $M_{2}$ between the left and right resonators at the carrier frequency $\omega_{c}$, which, for optimal frequency conversion, is set at the band centre of the right resonator. When operated from left to right, the circuit carries out the modulation of low-frequency signals of frequency $\omega_{0}$ travelling on ports $A$ and $B$ to generate sidebands at $\omega_{c} \pm \omega_{0}$ travelling on the high-frequency line $C$. It carries out the inverse operation of demodulation when operated in reverse from right to left. $\mathbf{b}$, Spectral density/response landscape for different spatial channels (or ports) of the UDC circuit in $\mathbf{a}$ as a function of frequency. The dotted lines represent the couplings between different ports. The solid and the dashed arrows represent different frequencies and respective conjugates. The resonance line shapes of the two spatially distinct ports $A$ and $B$ are centred at $\omega_{A}=\omega_{B}=1 / \sqrt{L_{A, B} C_{A, B}}$. Here we show the case when the incoming signal at $\omega_{0}$ is resonant with the centre frequency $\left(\omega_{0}=\omega_{A, B}\right)$. The two sidebands generated by the UDC on channel $C$ are detuned from the pump at $\omega_{\mathrm{c}}$ by equal amounts.

on low-noise microwave parametric amplifiers, which, with the exception of designs based on the current-biased d.c. superconducting quantum interference device (SQUID), are also operated in a reflection mode with both the input and output signals collected on the same spatial channel ${ }^{15,16}$. However, circulators (and isolators) routinely use bulk components made from ferrites to achieve non-reciprocal phase shifts (Fig. 1c) through Faraday rotation, making them unamenable to chip fabrication. Moreover, to bias the magnetic field in the ferrite, most of these devices use a permanent magnet, which may channel flux into the superconducting device under test.

Here, we present the full analysis of a model for a four-port circulator based on parametric active devices with no magnetic components. In active devices the energy source-provided by the pump-acts as the external 'bias' field and sets the reference phase for the system, in analogy with the role played by the magnetic field in a Faraday medium. We exploit this effect in a cascade of active devices with pump phases at each stage shifted appropriately to obtain non-reciprocal transmission.
The main building block of our design is a reversible in-phase/quadrature modulator capable of carrying out noiseless frequency up- and down-conversion. A convenient analytical model capturing the fundamental properties of the device is shown in Fig. 2a. The device comprises two low-frequency inductor-capacitor (LC) resonators (addressed by two semi-infinite transmission lines A and B) coupled to a high-frequency resonator (addressed by the transmission line $\mathrm{C}$ ) through time-varying couplings $M_{1}, M_{2}$ that emulate the role of the pump drive in active nonlinear devices and transfer energy from the tone at $\omega_{\mathrm{c}}$ to the signal modes propagating on the transmission lines. It operates in a manner analogous to the in-phase/quadrature modulation schemes routinely used in radiofrequency communication systems and microwave pulse engineering (hence the name) and converts two orthogonal spatial modes travelling on two distinct spatial ports $(\mathrm{A}, \mathrm{B})$ at the same frequency (here $\left.\omega_{0}\right)$ into two orthogonal temporal modes travelling on the same spatial line $(\mathrm{C})$ at different frequencies $\left(\omega_{+}, \omega_{-}\right)$. However, in view of the reversible and noiseless frequency conversion carried out by this device (Fig. 2a), we will henceforth refer to it as the up/down-converter (UDC). In practice, such a device can be implemented on-chip using two ring modulators based on Josephson junctions, along the lines of the recently demonstrated experiment with a Josephson parametric converter ${ }^{17,18}$.

The complete design for the active circulator (Fig. 3a) consists of a UDC functioning as a frequency up-converter, a phase shifter and a second UDC functioning as a frequency down-converter.

A concise representation of the dynamics at each of the three stages in the cascade is provided by the scattering matrix $S$ that relates the outgoing wave amplitudes to the incoming wave amplitudes as seen from the ports of a network. We begin by deriving the scattering matrix of the UDC stage and obtain (see the equations (5) and (6) of Methods section)

$$
\left(\begin{array}{c}
a_{0}{ }^{\prime \text { out }} \\
a_{0}{ }^{\text {out }} \\
b_{+}{ }^{\text {out }} \\
b_{-}{ }^{\text {tout }}
\end{array}\right)=\left(\begin{array}{cccc}
r_{0} & -q_{0} & t_{\mathrm{d}} \mathrm{e}^{-i \phi} & s_{\mathrm{d}} \mathrm{e}^{i \phi} \\
q_{0} & r_{0} & i t_{\mathrm{d}} \mathrm{e}^{-i \phi} & -i s_{\mathrm{d}} \mathrm{e}^{i \phi} \\
t_{\mathrm{u}} \mathrm{e}^{i \phi} & -i t_{\mathrm{u}} \mathrm{e}^{i \phi} & r_{+} & 0 \\
-s_{\mathrm{u}} \mathrm{e}^{-i \phi} & -i s_{\mathrm{u}} \mathrm{e}^{-i \phi} & 0 & r_{-}
\end{array}\right)\left(\begin{array}{c}
a_{0}{ }^{\prime \text { in }} \\
a_{0}{ }^{\prime \text { in }} \\
b_{+}{ }^{\text {in }} \\
b_{-}{ }^{\text {in }}
\end{array}\right)
$$

Here $a$ and $b$ denote the (reduced) amplitudes or the annihilation operators for the waves travelling on left and right transmission lines of the UDC respectively. This notation will be consistently used to denote the left- $(a)$ and right- $(b)$ propagating modes at each stage of the device hereafter (see Fig. 3a). These satisfy bosonic commutation relations of the form ${ }^{19}$

$$
\left[a_{i}^{\text {in/out }}, a_{j}^{\dagger, \text { in/out }}\right]=\delta\left(\omega_{i}-\omega_{j}\right)
$$

In writing equations (1) and (2), we have set $a_{0}=a\left[\omega_{0}\right], a_{+}=$ $a\left[\omega_{+}\right], a_{-}=a\left[\omega_{-}\right]$(see Fig. 2b). Similarly, the reflection coefficients at various ports are denoted by $r_{0}, r_{+}$and $r_{-}$. The cross reflection between the low-frequency signal ports is denoted by $q_{0}$. The transmission coefficients are written as $t$ (transmission without conjugation) and $s$ (transmission with conjugation) with subscripts $(\mathrm{u}, \mathrm{d})$ indicating the up-conversion and down-conversion, respectively. These scattering coefficients are a function of frequency in general (see Supplementary Information for a detailed derivation). It is useful to note that the phase of the carrier, denoted by $\phi$, affects only the transmitted amplitudes and rotates the two sidebands in opposite directions as can be seen from the corresponding scattering coefficients $s$ and $t$ in equation(1). The invariance of reflection amplitudes to the phase of the coupling will be important in understanding total reflections of the cascade, as we describe later.

Furthermore, we note that the matrix obtained in equation (1) is non-unitary, that is $S^{\dagger} S \neq 1$, which implies non-conservation 


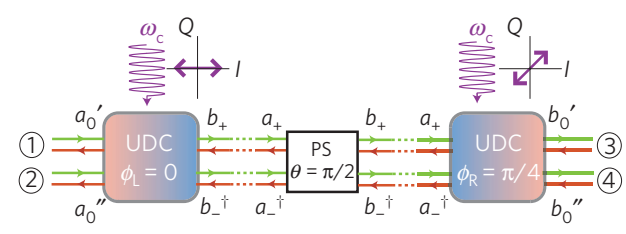

b

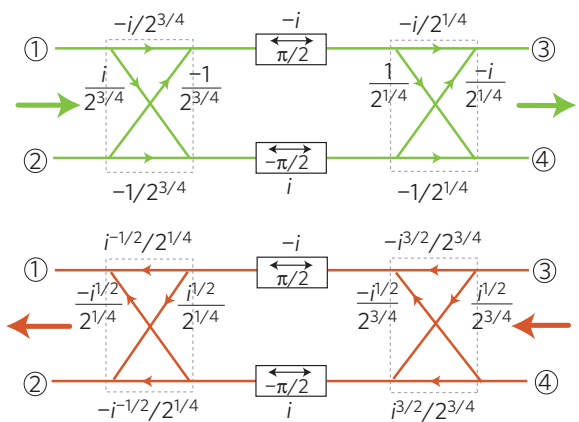

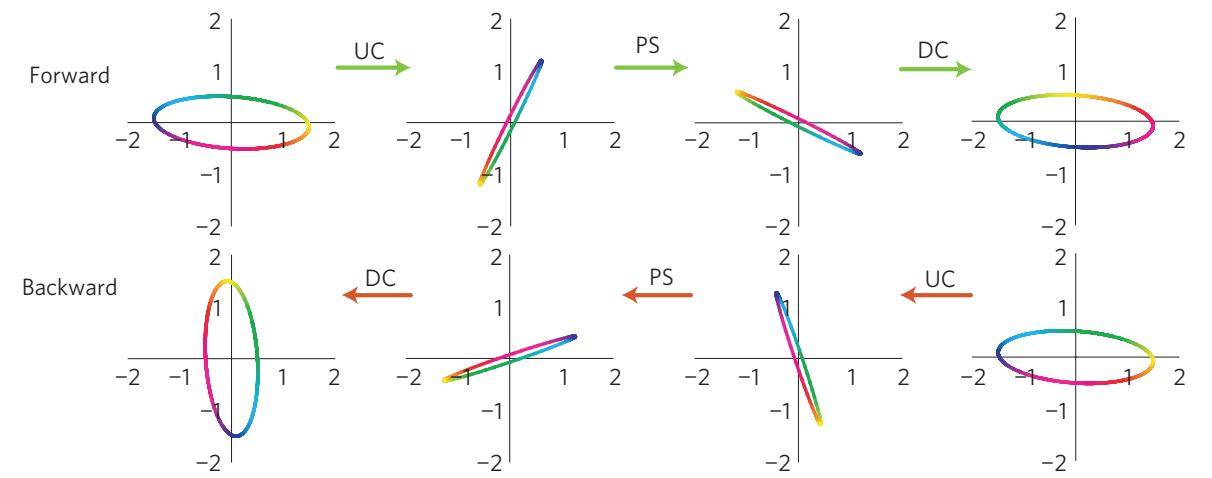

Figure 3 | Description of the active circulator. a, Circuit schematic of the active circulator design. The first UDC stage acts as a frequency up-converter (UC; also indicated by a gradation in the colour of the relevant square) with a parametric coupling modulated at the carrier frequency

$\omega_{\mathrm{C}}=\omega_{+}-\omega_{0}=\omega_{-}+\omega_{0}$ and a phase $\phi_{\mathrm{L}}=0$. This is followed by a phase shifter (PS) that phase shifts both the sidebands by $\pi / 2$, in opposite directions. They are then demodulated by the final UDC stage acting as a frequency down-converter (DC), with the pump phase $\phi_{\mathrm{R}}=\pi / 4$. $\mathbf{b}$, Forward ( $\left.g r e e n\right)$ and backward (red) propagation diagrams calculated using the transfer matrix method for a with an appropriate choice of detuning $\left(\delta_{ \pm}=1 / \sqrt{2}\right)$ and coupling strengths $\left(\alpha_{\mathrm{L}}=\alpha_{\mathrm{R}}=2^{-3 / 4}\right)$ for maximum isolation. $\mathbf{c}$, Representation of the device operation using modulation ellipses at each stage in the cascade. The top panel shows the forward propagation for the case when two distinct signals enter ports 1 and 2 respectively $\left(a_{0}{ }^{\prime}=1, a_{0}{ }^{\prime \prime}=0.5 \mathrm{e}^{i \eta} ; \eta=\pi / 24\right)$ and the

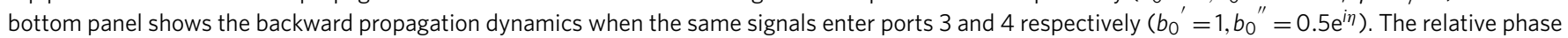
and amplitudes are chosen to represent the most general case of two input signals that differ in both amplitude and phase. The relative phase difference between the two signals is encoded as the tilt of the modulation ellipse in the in-phase/quadrature plane and their average phase is represented as the colour along the perimeter of the ellipse, with yellow indicating zero phase (also see Supplementary Information for more details).

of photon number as is natural for an active device. The matrix recovers its unitary form as we turn off the couplings $M_{1}, M_{2}$ responsible for energy transfer between the pump and the signal modes. The full $8 \times 8$ matrix (Supplementary Equation S17), describing the device operation for all modes and their respective conjugates, fulfils the fundamental requirement of symplecticity ${ }^{17,20}$.

We can similarly describe the action of the frequencyindependent phase-shifting stage using a scattering matrix of the form

$$
\left(\begin{array}{c}
a_{+}{ }^{\text {out }} \\
a_{-}{ }^{\text {tout }} \\
b_{+}{ }^{\text {out }} \\
b_{-}{ }^{\text {tout }}
\end{array}\right)=\left(\begin{array}{cccc}
0 & 0 & \mathrm{e}^{-i \theta} & 0 \\
0 & 0 & 0 & \mathrm{e}^{i \theta} \\
\mathrm{e}^{-i \theta} & 0 & 0 & 0 \\
0 & \mathrm{e}^{i \theta} & 0 & 0
\end{array}\right)\left(\begin{array}{c}
a_{+}{ }^{\text {in }} \\
a_{-}{ }^{\text {in }} \\
b_{+}{ }^{\text {in }} \\
b_{-}{ }^{\text {in }}
\end{array}\right)
$$

The scattering matrix of the whole device is then obtained using the transfer matrix formulation, as described in the Methods section (also see Supplementary Information).

In our analysis we consider the operation at resonance, that is, when the input signal frequency coincides with the band centre of the input resonators. Setting the phase of the pump at the first UDC stage $\phi_{\mathrm{L}}=0$ for calculational simplicity, we observe a transmission resonance for $\theta= \pm \pi / 2$ (phase rotation by the phase shifting stage), $\phi_{\mathrm{R}}=\pi / 4$ (phase of the pump at the second UDC stage), $\delta_{ \pm}=1 / \sqrt{2}$ (detuning of the sidebands from the pump in units of linewidth, that is, half-width at full-maximum of the resonance line shape) of the high-frequency resonator) and $\alpha_{\mathrm{L}}=\alpha_{\mathrm{R}}=M_{0} / \sqrt{L_{\mathrm{A}, \mathrm{B}} L_{\mathrm{C}}}=2^{-3 / 4}$ (strength of the parametric coupling). For this choice of parameters, we obtain the scattering matrix of the complete device as

$$
S_{\text {total }}=\left(\begin{array}{cccc}
0 & 0 & 0 & i \\
0 & 0 & -i & 0 \\
i & 0 & 0 & 0 \\
0 & i & 0 & 0
\end{array}\right)
$$

This is the matrix of a perfect four-port circulator. The analogy between a conventional circulator and the active circulator design proposed in this Letter is made apparent from the respective wave propagation diagrams in Figs $1 \mathrm{c}$ and $3 \mathrm{~b}$ (see Supplementary Information for details on the calculation of coefficients on different arms in Fig. 3b). Nonetheless there are important differences between the two designs despite the identity of the final $S$ matrix. The coefficients on the forward- (green) and backward- (red) propagating arms of the active circulator design (Fig. 3b) involve deamplification followed by amplification, unlike the passive splitters $\left(90^{\circ}\right.$ or $180^{\circ}$ hybrids) employed in Faraday rotation schemes. This can be observed by squaring the amplitudes on each of the two arms originating from (or terminating into) a port and calculating the net power output, for each isolated UDC stage. It is straightforward to observe that, unlike the case of Fig. 1c, they do not add up to unity. The overall transmission, however, is still unity owing to an exact cancellation of the larger and smaller than unity gains. The wave propagation diagrams in Fig. $3 \mathrm{~b}$ reveal another important difference of this design from that of a conventional 


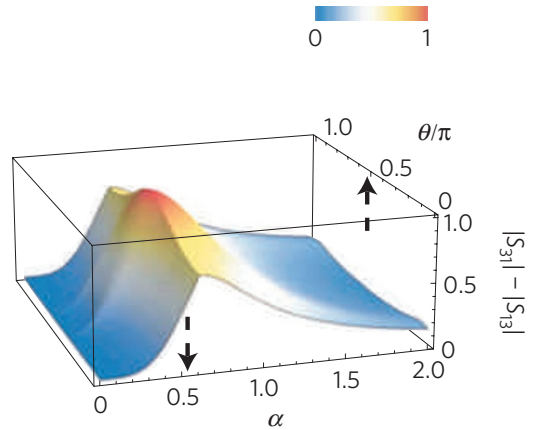

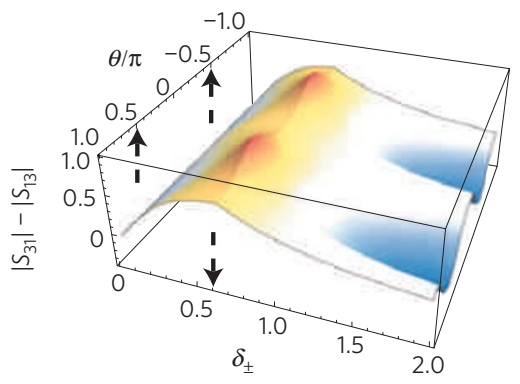

C

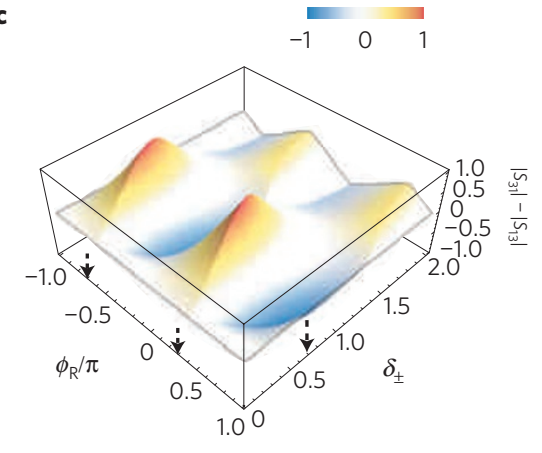

Figure 4 | Variation of the difference between forward and backward transmission coefficients ( $\left.\left|\mathbf{s}_{\mathbf{3 1}}\right|-\left|\mathbf{s}_{\mathbf{1 3}}\right|\right)$. a-c, Asymmetry in transmission calculated as a function of strength of the coupling $\alpha$ and phase rotation $\theta$ carried out by the second phase-shifting stage (a), detuning $\delta_{ \pm}$of the sidebands from the pump and phase rotation $\theta$ (b), and detuning $\delta_{ \pm}$and the phase of the pump at second UDC stage $\phi_{\mathrm{R}}$ (c). The points of maxima correspond to the ideal values reported in the text. The plot in $\mathbf{b}$ also shows the periodicity of the response of the device as a function of $\theta$. In $\mathbf{c}$, the variation with respect to the pump phase shows the reversal of transmission characteristics with $\phi_{\mathrm{R}} \rightarrow-\phi_{\mathrm{R}}$. As in $\mathbf{b}$, the response is periodic in $\phi_{\mathrm{R}}$ with a period equal to $\pi$. It can be seen that the design continues to work for moderate deviations from the preferred phase angle $\theta=\pi / 2$, coupling $\alpha_{\mathrm{L}}=\alpha_{\mathrm{R}}=2^{-3 / 4}$, detuning $\delta_{ \pm}=1 / \sqrt{2}$ and pump phase $\phi_{R}=\pi / 4$ (values indicated with dashed arrows along the axis).

circulator. The non-reciprocal action of the active circulator is not based on any non-reciprocal phase shifters; instead it relies on the active stages used for frequency up- and down-conversion. The phase-matching condition in the forward direction is met by tuning the phase of the coupling at the input and output UDC stages. In the reverse direction, the phase mismatch leads to unity transmission in the spatially orthogonal port instead, leading to complete isolation between the incident signal port and its corresponding output port. Figure $3 \mathrm{c}$ shows a convenient method to visualize this circulator action geometrically by mapping the device dynamics at different stages using a modulation ellipse representation (see the Methods section and Supplementary Fig. S2 for details).

Furthermore, as seen from equation (1), the reflection coefficients at the UDC stages are non-zero for all modes. However, for the whole cascade, the total reflection is identically zero at every port $\left(s_{i i}=0\right.$ for all $i$ in equation (4)). This remarkable cancellation of total reflections for the cascade can be understood in analogy with a Fabry-Perot resonance where a cavity flanked by two identical reflecting mirrors exhibits unity transmission at resonance. The total phase shift between the active 'mirrors' in our device: $(\pi / 2)_{a_{+}}-(-\pi / 2)_{a_{-} \dagger}=\pi$, is akin to the resonance condition when a half-wavelength of the incident radiation equals the length of the Fabry-Perot cavity. Also the reflections at the two UDC stages are identical (as the reflection coefficients are independent of the phase angle $\phi$, equation (1)), fulfilling the second condition for the transmission resonance and net cancellation of reflections. A point of distinction between the two pictures is that the symmetry of the active circulator design is described by a subgroup of the $S U(4)$ group (the group formed by $4 \times 4$ complex matrices of unit determinant, equation (4)), and not by the $S U(2)$ group that describes passive lossless two-port devices such as the Fabry-Perot resonator.

We note a recent theoretical paper ${ }^{21}$ that also showed the existence of noiseless non-reciprocity in a nonlinear circuit involving microwave resonators coupled through Josephson junctions. Although this proposal is based on a passive rather than active Josephson circuit, unlike our proposal, it involves very small Josephson junctions susceptible to offset charges. Also, owing to its low characteristic energy, it handles qubit readout signals with a lower dynamic range than our proposed device. Furthermore, the dependence of the action of our active circulator design on different parameters (Fig. 4) shows that the isolation achieved is robust to reasonable deviations of parameters from their ideal values; thus, the active circulator design holds promise for use in practical circuits. Another interesting feature of our device is the reversal of its transmission characteristics with the phase of the pumps $\left(\phi_{\mathrm{L}, \mathrm{R}} \mapsto-\phi_{\mathrm{L}, \mathrm{R}}\right)$ (Fig. 4c). In the classic circulators based on passive Faraday rotation, this can be accomplished by changing the polarity of the magnetic bias field. Thus, the relative shift of the pump phase in an active device indeed plays a role equivalent to the magnetic field in a Faraday medium.

Our scheme for non-reciprocal wave propagation relies on the directionality imposed by shifting the phases of the pump drives in a cascade of parametrically active stages. The proposed design operates noiselessly as the complete device has unity gain and consists of purely dispersive components with no dissipation, making it attractive for quantum information applications using superconducting circuits ${ }^{22}$. As the proposed protocol is generic in nature, it should be easily adaptable to optical frequencies where it can complement the recently proposed designs of non-reciprocal light propagation based on dynamical modulation of the refractive index of photonic structures ${ }^{23}$ and the use of a surface waveguide on photonic crystals ${ }^{24}$. In addition to providing a design for practical on-chip non-reciprocal devices, the treatment described in this Letter may also give theoretical insights into the inherently directional dynamics of devices such as the microwave d.c. SQUID (ref. 25), when additional active stages are included in the chain. This would be useful in tackling unanswered questions pertaining to the quantum noise of d.c. SQUID amplifiers ${ }^{26}$.

\section{Methods}

The scattering matrix for the UDC is calculated from the network impedance matrix $Z$, as seen from its ports, using the identity

$$
S=\left(Z+Z_{0}\right)^{-1} \times\left(Z-Z_{0}\right)
$$

where

$$
Z_{0}=\operatorname{diag}\left(Z_{\mathrm{A}}, Z_{\mathrm{B}}, Z_{\mathrm{C}}, Z_{\mathrm{C}}\right)
$$

Here, the symbol $\times$ denotes matrix multiplication and $Z_{\mathrm{A}}=Z_{\mathrm{B}}, Z_{\mathrm{C}}$ are the characteristic impedances of the semi-infinite transmission lines serving as lowand high-frequency ports respectively.

For each stage of the cascade, we now transform from the scattering matrix, which provides a port-neutral relation between various input and output ports, to a port-specific transfer matrix:

$$
\left(\begin{array}{c}
a_{+}{ }^{\text {out }} \\
a_{-}{ }^{\text {tout }} \\
b_{+}{ }^{\text {out }} \\
b_{-}{ }^{\text {tout }}
\end{array}\right)=S\left(\begin{array}{c}
a_{+}{ }^{\text {in }} \\
a_{-}{ }^{\text {in }} \\
b_{+}{ }_{\text {in }} \\
b_{-}{ }_{\text {†i }}
\end{array}\right) \mapsto\left(\begin{array}{c}
b_{+}{ }^{\text {out }} \\
b_{+}{ }^{\text {in }} \\
b_{-}{ }^{\text {tout }} \\
b_{-}{ }^{\text {tin }}
\end{array}\right)=T\left(\begin{array}{c}
a_{+}{ }^{\text {in }} \\
a_{+}{ }^{\text {out }} \\
a_{-}{ }^{\text {tin }} \\
a_{-}{ }^{\text {tout }}
\end{array}\right)
$$

The transfer matrix representation ${ }^{6}$ is especially useful for analysing the proposed design, because it is straightforward to calculate the total transfer 
matrix of the device by multiplying the respective transfer matrices of different stages in the cascade,

$$
T_{\text {total }}=T_{\mathrm{DC}_{\mathrm{R}}} \times T_{\mathrm{PS}} \times T_{\mathrm{UC}_{\mathrm{L}}}
$$

Here the subscripts L, R index the left-hand upconversion (UC) and right-hand downconversion (DC) stage and the subscript PS refers to the intermediate phase shifter (Fig. 3a). The scattering matrix of the whole device is obtained from $T_{\text {total }}$ using the inverse of the transformation in equation (7). For the purpose of evaluating $T_{\text {total }}$ from equation (8), $T_{\mathrm{UC}}, T_{\mathrm{DC}}$ and $T_{\mathrm{PS}}$ are calculated from equations (1) and (3) respectively, while noting that

$$
\begin{aligned}
T_{\mathrm{DC}} & =F^{-1} \times T_{\mathrm{UC}}^{-1} \times F \\
& =F \times T_{\mathrm{UC}}^{-1} \times F, \quad\left(F^{-1}=F\right)
\end{aligned}
$$

In equation (9), $F=\sigma_{X} \otimes I_{2}$ ( $\sigma_{X}$ is the two-dimensional Pauli spin matrix and $I_{2}$ is the two-dimensional unity matrix). This matrix $F$ is required to flip the indices, thus maintaining consistency in labelling the 'in' and 'out' amplitudes along a given direction of propagation (see Supplementary Information for additional details).

The modulation ellipses in Fig. $3 \mathrm{c}$ are calculated using the transfer matrices, with coefficients indicated in Fig. 3b. This representation is inspired by the polarization ellipse used to represent the state of polarization of an electromagnetic wave (linear, circular or elliptical), which involves recording the trajectory traced out by the tip of the polarization vector of light (defined by the instantaneous direction of the electric field vector $\mathbf{E}$ ) in a plane perpendicular to the direction of propagation. Equivalently, a two-dimensional representation of the components $E_{X}$ and $E_{Y}$ of the electric field in the complex plane can be used to obtain a geometric description of the polarization of the light wave. In the case of a modulation ellipse representation of Fig. $3 c$, we extend this idea to map two distinct orthogonal modes $(x, y)$ at each stage of the proposed device (spatial: $\left(x=a_{0}^{\prime}, y=a_{0}^{\prime \prime}\right)$ or temporal: $\left.\left(x=a_{+}, y=a_{-}\right)\right)$as an ellipse in the plane defined by the coordinates $I=\operatorname{Re}[x+y], Q=\operatorname{Im}\left[x-y^{*}\right]$. This exercise shows that the final ellipses obtained at the output in the cases of forward and backward propagation through the device are rotated by $90^{\circ}$ with respect to each other, indicating that in the case of reverse propagation the orthogonal spatial port, relative to the forward propagation, receives the transmitted energy, leading to a circulator action.

Received 19 July 2010; accepted 18 November 2010; published online 30 January 2011

\section{References}

1. Schoelkopf, R. J., Wahlgren, P., Kozhevnikov, A. A., Delsing, P. \& Prober, D. P. The radio-frequency single-electrin transistor (RF-SET): A fast and ultrasensitive electrometer. Science 280, 1238-1242 (1998).

2. Siddiqi, I. et al. RF-driven Josephson bifurcation amplifier for quantum measurement. Phys. Rev. Lett. 93, 207002 (2004).

3. Lupascu, A. et al. Quantum non-demolition measurement of a superconducting two-level system. Nature Phys. 3, 119-125 (2007).

4. Regal, C. A., Teufel, J. D. \& Lehnert, K. W. Measuring nanomechanical motion with a microwave cavity interferometer. Nature Phys. 4, 555-560 (2008).

5. Mallet, F. et al. Single-shot qubit readout in circuit quantum electrodynamics. Nature Phys. 5, 791-795 (2009).

6. Pozar, David M. Microwave Engineering 3rd edn, 471-482 (Wiley, 2005).

7. Van Harlingen, D. J. et al. Decoherence in Josephson-junction qubits due to critical-current fluctuations. Phys. Rev. B 70, 064517 (2004).

8. Makhlin, Y., Schön, G. \& Shnirman, A. Quantum-state engineering with Josephson-junction devices. Rev. Mod. Phys. 73, 357-400 (2001).
9. Schuster, A. An Introduction to the Theory of Optics 41-45 (Edward Arnold, 1904).

10. Barron, L. D. Parity and optical activity. Nature 238, 17-19 (1972).

11. Potton, R. J. Reciprocity in optics. Rep. Prog. Phys. 67, 717-754 (2004).

12. Ladd, T. D. et al. Quantum computers. Nature 464, 45-53 (2010).

13. Clarke, J. \& Wilhelm, F. K. Superconducting quantum bits. Nature 453, 1031-1042 (2008)

14. Wallraff, A. et al. Strong coupling of a single photon to a superconducting qubit using circuit quantum electrodynamics. Nature 431, 162-167 (2004).

15. Castellanos-Beltran, M. A. \& Lehnert, K. W. Widely tunable parametric amplifier based on a superconducting quantum interference device array resonator. Appl. Phys. Lett. 91, 083509 (2007).

16. Yamamoto, T. et al. Flux-driven Josephson parametric amplifier. Appl. Phys. Lett. 93, 042510 (2008).

17. Bergeal, N. et al. Analog information processing at the quantum limit with a Josephson ring modulator. Nature Phys. 6, 296-302 (2010).

18. Bergeal, N. et al. Phase preserving amplification near the quantum limit with a Josephson ring modulator. Nature 465, 64-68 (2010).

19. Yurke, B. in Input Output Theory in Quantum Squeezing (eds Drummond, P. D. \& Ficek, Z.) 53-95 (Springer, 2004).

20. Kamal, A., Marblestone, A. \& Devoret, M. H. Signal-to-pump back action and self-oscillation in double-pump Josephson parametric amplifier. Phys. Rev. B 79, 184301 (2009).

21. Koch, J., Houck, A. A., Le Hur, K. \& Girvin, S. M. Time-reversal-symmetry breaking in circuit-QED-based photon lattices. Phys. Rev. A 82, 043811 (2010).

22. DiCarlo, L. et al. Demonstration of two-qubit algorithms with a superconducting quantum processor. Nature 460, 240-244 (2009).

23. Yu, Z. \& Fan, S. Complete optical isolation created by indirect interband photonic transitions. Nature Photon. 3, 91-94 (2009).

24. Liu, A. Q., Khoo, E. H., Cheng, T. H., Li, E. P. \& Li, J. A frequency-selective circulator via mode coupling between surface waveguide and resonators. Appl. Phys. Lett. 92, 021119 (2008).

25. Clarke, J. \& Braginsky, A. I. (eds) The SQUID Handbook Vol. I Fundamentals and Technology of SQUIDs and SQUID Systems (Wiley-VCH Verlag GmbH and Co. KGaA, 2004).

26. Clarke, J. \& Braginsky, A. I. (eds) The SQUID Handbook Vol. II Applications of SQUIDs and SQUID Systems (Wiley-VCH Verlag GmbH and Co. KGaA, 2006).

\section{Acknowledgements}

We acknowledge useful discussions with S. M. Girvin, J. Koch, L. Spietz and R. J. Schoelkopf. This research was supported by the US National Security Agency through the US Army Research Office grant W911NF-05-01-0365, the W. M. Keck Foundation, the US National Science Foundation through grant DMR-0653377 (A.K. and M.H.D.) as well as by the Office of the Director of National Intelligence (ODNI), Intelligence Advanced Research Projects Activity (IARPA), through the Army Research Office. All statements of fact, opinion or conclusions contained herein are those of the authors and should not be construed as representing the official views or policies of IARPA, the ODNI or the US Government (J.C.). M.H.D. also acknowledges partial support from the College de France and the French Agence Nationale de la Recherche.

\section{Author contributions}

A.K. is a graduate student and J.C. and M.H.D. are the principal investigators on the project.

\section{Additional information}

The authors declare no competing financial interests. Supplementary information accompanies this paper on www.nature.com/naturephysics. Reprints and permissions information is available online at http://npg.nature.com/reprintsandpermissions. Correspondence and requests for materials should be addressed to M.H.D. 